\title{
Characterisation of discharge events during plasma electrolytic oxidation
}

\author{
C.S. Dunleavy ${ }^{\mathrm{a}}$, I.O. Golosnoy ${ }^{\mathrm{b}}$, J.A. Curran ${ }^{\mathrm{a}, \mathrm{c}}$, T.W. Clyne ${ }^{\mathrm{a}, *}$ \\ a Department of Materials Science \& Metallurgy, Cambridge University, Pembroke Street, Cambridge, CB2 3QZ, UK \\ b School of Electronics and Computer Science, University of Southampton, SO17 1BJ, UK \\ c Keronite International Ltd., Granta Park, Great Abington, Cambridge, CB21 6GP, UK
}

\section{A R T I C L E I N F O}

Article history:

Received 12 March 2009

Accepted in revised form 5 May 2009

Available online 12 May 2009

\section{Keywords:}

Plasma electrolytic oxidation

Micro-arc oxidation

Spectral analysis

Electrical breakdown

Coatings

\begin{abstract}
A B S T R A C T
A study has been made of the electrical characteristics and optical emission spectra exhibited when discharge events take place during plasma electrolytic oxidation processing. Both conventional and small area experimental arrangements have been employed, allowing detailed measurement of durations, and temporal distributions, as well as such characteristics as charge transfer, and power. Individual discharges are of short duration, typically tens to hundreds of microseconds, but there is a strong tendency for them to occur in cascades that commonly last between several $\mathrm{ms}$ and several tens of ms. The composition, temperature and electron density of the plasma formed during PEO processing are inferred from characteristics of the emission spectra. This confirms that there are two distinct regions of plasma; a lower density peripheral region at $~ 3500 \mathrm{~K}$, and a higher density core at $\sim 16,000 \pm 3500 \mathrm{~K}$. The implications of these results are considered in terms of the interpretation of different types of experimental measurement, and attention is also briefly given to how such behaviour might relate to the mechanisms of growth.
\end{abstract}

(c) 2009 Elsevier B.V. All rights reserved.

\section{Introduction}

Plasma Electrolytic Oxidation (PEO), also known as Micro Arc Oxidation (MAO), Spark Anodising and Microplasma Oxidation, is a processing technique in which the surfaces of metals such as aluminium, magnesium and titanium are converted into oxide coatings. These coatings can range from tens to hundreds of microns in thickness, depending on the power supply, substrate and electrolyte used. The process is known to involve large numbers of short-lived sparks (electrical discharges), caused by localized electrical breakdown of the growing coating. These discharges clearly play an important role in the coating growth mechanism. They leave characteristic "craters" on the free surface of the growing coating. Sundararajan and Rama Krishna [1] found that the average diameter of such craters (on $7075 \mathrm{Al}$ ) increased from $1.3 \mu \mathrm{m}$ after $1 \mathrm{~min}$ to $2.4 \mu \mathrm{m}$ after $30 \mathrm{~min}$. The surface roughness also increased as the coating thickened. The areal density of these craters, on the other hand, has been reported to decrease substantially with increasing process time [1,2]. Matykina et al. [3] reported a similar effect with PEO coatings on Ti, although in that case the reduction was less marked. Also likely to be relevant is the report [4] that PEO coatings contain a fine, inter-connected porosity network, which appears to extend throughout the thickness, or at least most of it.

Optical emission spectroscopy has been applied previously to PEO coatings. The first investigation of this type was carried out in 1994 by Klapkiv et al. [5], concerning discharges during PEO processing of Al. A further study on Al was carried out in 2005 by Mécuson et al. [6],

\footnotetext{
* Corresponding author.

E-mail address: twc10@cam.ac.uk (T.W. Clyne).
}

while Arrabal et al. [7] recently studied a Mg alloy in this way. Of these three investigations, only that of Klapkiv et al. was carried out using a spectrograph with sufficient resolution to allow estimates of the plasma electron temperature to be made from broadening of spectral lines. They estimated typical plasma temperatures to be 6800 $7800 \mathrm{~K}$. The physical properties of the plasma within the discharge were investigated further by Klapkiv in a subsequent paper [8] and the chemical reactions taking place within a discharge, and the internal structure of the plasma, were also later discussed by him [9]. The plasma structure proposed by Klapkiv comprises a central core, at $\sim 7000 \mathrm{~K}$, containing free electrons, ionic and atomic species, surrounded by lower temperature regions, in which different plasma components and reactions are found, depending on the temperature. The thermodynamics, and expected yields of reaction products, in such discharge channels (through PEO coatings on $\mathrm{Al}, \mathrm{Mg}$ and $\mathrm{Zr}$ ) were subsequently examined by Klapkiv et al. [10], who also discussed possible reaction pathways to the final products.

Optical characteristics of individual discharges have also been studied via (digital) video recording. For example, Yerokhin et al. [11] estimated the apparent size of discharges from study of $24 \mathrm{~Hz}$ video recordings made during $50 \mathrm{~Hz}$ AC PEO processing of Al. They also estimated the total area fraction apparently undergoing discharge (at any given time). They estimated the discharge durations required to provide sufficient heat to stimulate $\gamma-\alpha$ transitions in alumina to be between 0.25 and $3.5 \mathrm{~ms}$. Mécuson et al. [6] also recorded video images, with a time resolution of $2 \mathrm{~ms}$, but did not draw any inferences about discharge lifetimes. Matykina et al. [3] recorded video images during PEO processing of Ti, using an exposure period of $10 \mathrm{~ms}$. They produced probability density plots of apparent discharge lifetime 
and diameter. The lifetimes of events were reported to vary from less than $40 \mathrm{~ms}$ to over $600 \mathrm{~ms}$. The areal density of discharges was also investigated, as a function of time. However, when they compared this to the number of craters visible on the surface after processing, they found a discrepancy of two orders of magnitude. Arrabal et al. [7] recently used high speed photography (exposure period $\sim 50 \mathrm{~ms}$ ) to examine discharges during PEO processing of Mg. They found event lifetimes to range from $0.05 \mathrm{~ms}$ up to $4 \mathrm{~ms}$.

Study of the electrical characteristics of PEO processing dates back further than spectral or optical studies. The work of Van et al. [12], carried out in 1977, involved recording the currents corresponding to single sparks. They found the largest current pulses to be $\sim 70 \mathrm{~mA}$, and durations up to $\sim 170 \mu \mathrm{s}$. One concern with this work is that the material used for single spark measurements was stainless steel. This material is not suitable for conventional PEO processing, so that the results may not be directly relevant to most of the work being described here. Electrical breakdown during the final stages of anodizing of Ta and Al was studied by Kadary and Klein [13] and Klein et al. [14]. These studies were largely concerned with the rate of electrical breakdown, as a function of applied voltage and anodizing current density. However, the magnitude of the breakdown currents was found [13] to be in the mA range. Such currents are much lower than those generally observed during PEO processing, probably because they correspond to the very first sparks seen in the early stages of the PEO process. The initial breakdown process is thought to be via electron injection into the coating, with a subsequent electron avalanche leading to breakdown. Such mechanisms are widely assumed to be operative during electrical breakdown of wide bandgap insulators, such as alumina, and have been the subject of numerous experimental [15-17] and theoretical [15,18-21] investigations.

Whether such a mechanism occurs during electrical breakdown in PEO coatings is not clear, especially since the fields required for such mechanisms are of the order of MV $\mathrm{cm}^{-1}$, whereas the average field across a growing PEO coating quickly falls well below these levels. It's also well-established that the voltage needed for continued dielectric breakdown to occur during PEO processing does not scale linearly with coating thickness, and indeed changes little after an initial sharp rise. The explanation for this may be related to the fact that PEO coatings contain high levels of surface-connected porosity, reported by Curran and Clyne [4] as being 15-20\%. Also, TEM observations by Nie et al. [22] and Monfort et al. [23] on Al, and Arrabal et al. [7] on Mg, included reports of a thin, and apparently continuous layer, of amorphous material at the boundary with the substrate. These studies all reported that this layer has a thickness in the range from a few hundred nm to $1 \mu \mathrm{m}$.

The present work is partly aimed at producing a clear set of conclusions about the plasmas produced during electrical breakdown of PEO coatings on aluminium, particularly temperatures and lifetimes, and partly at obtaining improved understanding of the electrical characteristics of the breakdowns giving rise to these plasmas. This is done mainly with a customised small area system, in which features of single discharge events can be isolated and studied. Perfect correlation between measurements made with this system and the characteristics of discharges occurring during the PEO process itself is not possible, given the differences in experimental setup required. However, it will be shown that the small area studies are relevant to the industrial process by comparing the electrical characteristics of events produced during both types of processing.

\section{Experimental procedures}

\subsection{Coating production and monitoring of electrical characteristics}

Specimens of uniform thickness were produced on $40 \times 40 \times 3 \mathrm{~mm}$ coupons of AA6082, using a commercial $10 \mathrm{~kW}, 50 \mathrm{~Hz}$ AC processing facility. The initial RMS current density was $1500 \mathrm{~A} \mathrm{~m}^{-2}$ and samples were processed for 20 min each. All specimens were ground with 500 grade $\mathrm{SiC}$ paper, prior to processing.

The electrical circuit employed is shown in Fig. 1(a). The electrolyte used was a commercially-available product, with the approximate composition: 3-5 $\mathrm{g} \mathrm{l}^{-1}$ tetra-sodium pyrophosphate $\left(\mathrm{Na}_{4} \mathrm{P}_{2} \mathrm{O}_{7}\right), 3-5 \mathrm{~g} \mathrm{l}^{-1}$ sodium silicate $\left(\mathrm{Na}_{2} \mathrm{SiO}_{3}\right)$ and $1-2 \mathrm{~g} \mathrm{l}^{-1}$ potassium hydroxide $(\mathrm{KOH})$. The electrolyte had a conductivity of $6.5 \mathrm{mS} \mathrm{cm}^{-1}$ and a $\mathrm{pH}$ of 12 . Current and voltage were recorded continuously for the $20 \mathrm{~min}$ of processing, sampling every $120 \mu \mathrm{s}$, to provide about 166 data points per voltage cycle. After processing, the specimens were rinsed under running water for $3 \mathrm{~min}$, to prevent deposition of electrolyte components during drying. Coating thickness was measured using an Eban 2000 MKII eddy current thickness gauge.

\subsection{Small area $D C$ testing}

Individual electrical discharge events were studied using the electrical circuit shown in Fig. 1(b), with the coating area exposed to electrolyte being $\sim 28 \mathrm{~mm}^{2}$ (circle of $6 \mathrm{~mm}$ diameter) and the electrolyte volume being $\sim 280 \mathrm{~mm}^{3}$. This technique is based on the work of Klein and co-workers $[13,14]$, with suitable modifications to allow for generation of electrical breakdowns through the thicker coatings generated by PEO processing. The power converter used to produce high voltage was a Hitek power DC-DC converter, which converts $0-12 \mathrm{~V}$ DC input to a DC output in the range $0-1500 \mathrm{~V}$. The output is linearly related to input, for inputs greater than $1.2 \mathrm{~V}$. The converter has a maximum continuous power output of $1.5 \mathrm{~W}$.

(a)

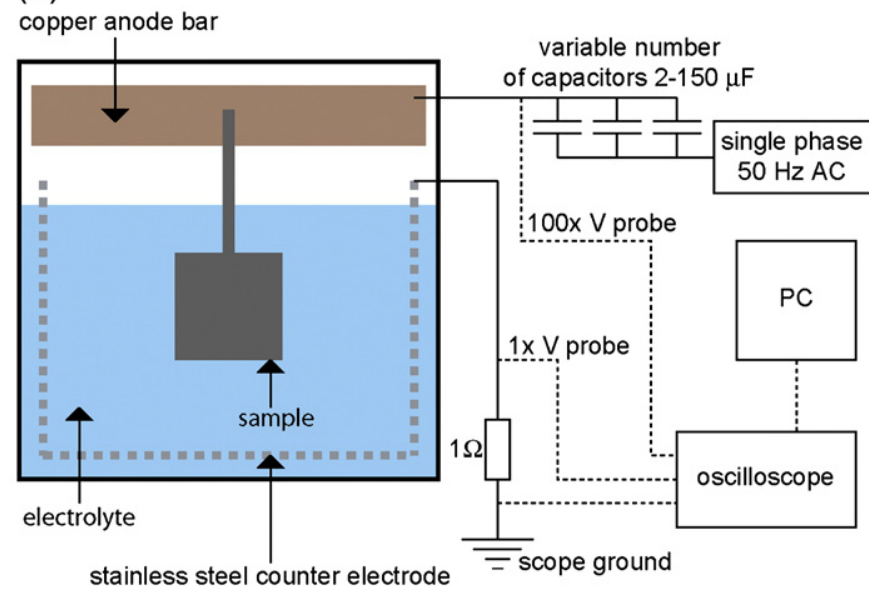

(b)

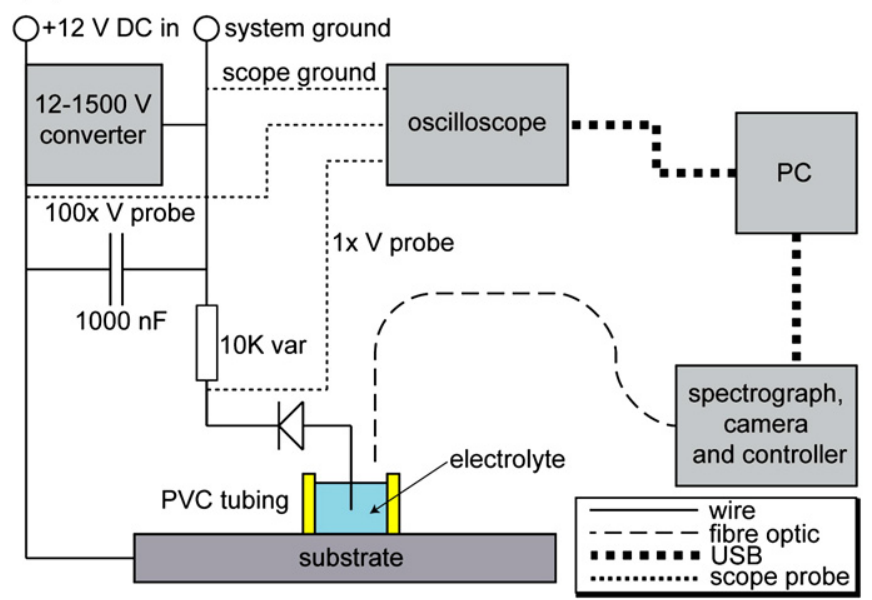

Fig. 1. Electrical circuits for (a) PEO coating production and (b) small area discharge study. 
The applied DC voltage increases until dielectric breakdown of the coating occurs. The nature of the output from the converter, and the small specimen area, were chosen so as to favour single discharge events. After an event initiates, the voltage decreases, due to the additional current loading. However, the capacitor in parallel with the sample (Fig. 1(b)) stores charge and energy, so that a series of discharges can be sustained. The resistor allows the current through the sample to be monitored. In order to maximise the voltage signal across the resistor in the range $0-20 \mathrm{~V}$, a value of $488 \Omega$ was found to be optimal. While it is clear that the electrical conditions during these tests are not identical to those in AC PEO processing of macroscopic components, it can be shown that the characteristics of events in the two situations are similar (see below).

Voltage monitoring was carried out with a $100 \times$ voltage attenuation probe, and the current was recorded using a $1 \times$ voltage probe. Voltage was logged using a Picoscope 5203 two-channel PC USB oscilloscope. Current and voltage histories were used to obtain the duration, initiation voltage, peak current, peak power, energy and charge transfer associated with individual discharge events. A large number of these $(\sim 350,000$, from 5 test wells) were studied, in order to obtain a good insight into statistical characteristics. Current and voltage were recorded at a sampling rate of $1 \mathrm{MHz}$, for periods of $10 \mathrm{~s}$ at a time. Longer times were logged as a series of $10 \mathrm{~s}$ windows, with the oscilloscope memory buffer transferred to the PC after each $10 \mathrm{~s}$ period. The maximum period of logging on any test well was $40 \mathrm{~s}$. This period was selected in view of the fact that, between 60 and $100 \mathrm{~s}$ into testing, significant changes started to appear in the current response of the samples, and data could no longer be considered representative of normal PEO coatings.

\subsection{In-situ discharge monitoring during $50 \mathrm{~Hz}$ AC processing}

To verify the relevance of the above $\mathrm{DC}$ work to conventional AC PEO processing, in situ measurements were made during the $50 \mathrm{~Hz} \mathrm{AC}$ processing of $\mathrm{Al}$ samples, by monitoring the current passing through a small area, processed in parallel with a larger area. The current passing through the small area was monitored using a Tektronix P6021 current probe. The main area processed was a circle of $30 \mathrm{~mm}$ diameter and the smaller area was a circle of $500 \mu \mathrm{m}$ diameter. Both were embedded in the same block of epoxy, so that only the quoted areas were exposed to the electrolyte. This parallel processing set up was selected because the $10 \mathrm{~kW}$ industrial supply used in this work cannot operate below about 0.3 A peak during each cycle, so the appropriate current density cannot be produced on a small sample area unless it represents a small fraction of the total area being processed. The voltage between the counter-electrode and the sample, and the current through the small area, were the variables measured, and this was done for $10 \mathrm{~s}$ segments at $1 \mathrm{MHz}$ sampling rate, as with the small area DC testing.

\subsection{Photomultiplier tube measurements}

Since a primary objective of this work is to correlate optical emission of the plasmas caused by discharges through PEO coatings with the electrical properties of these discharges, it is important to confirm that the optical emissions correlate with the current pulses recorded. This was accomplished using a Hamamatsu photonics H8249-102 photomultiplier tube module, with spectral range $185-900 \mathrm{~nm}$ and a $200 \mathrm{kHz}$ amplifier. The signal from the photomultiplier module was monitored concurrently with the current during small area DC testing. The signals were recorded at a lower sampling rate of $200 \mathrm{kHz}$ (every $5 \mu \mathrm{s}$ ), due to bandwidth limitations of the PMT module.

\subsection{Analysis of plasma emission spectra}

Spectra were acquired using a Princeton Instruments Acton SP300i spectrograph, with an 1800 line grating and PIXIS camera system
( 1340 by 400 pixels). Each spectrum had a wavelength range of $21.38 \mathrm{~nm}$ either side of the central wavelength, and the resolution was $0.08 \mathrm{~nm}$ at $280 \mathrm{~nm}$ centre wavelength, $0.079 \mathrm{~nm}$ at $310 \mathrm{~nm}$ and $0.074 \mathrm{~nm}$ at $395 \mathrm{~nm}$. Some wide range spectra were also taken, using a 300 line grating and $10 \mathrm{~s}$ accumulation time. These spectra had a relatively poor resolution of $0.5 \mathrm{~nm}$, and hence are unsuitable for accurate peak assignments. They were performed in order to locate the positions of peaks for high resolution measurements. The spectra were obtained using the set-up for small area DC testing (Fig. 1(b)). Atomic and ionic lines were identified using the NIST online spectral database [24] and the CRC Handbook [25].

\section{Observed process characteristics}

\subsection{Global current and voltage characteristics during $50 \mathrm{~Hz}$ AC processing}

The peak positive and negative voltage and current during each cycle, obtained from the data logged during processing, are presented in Fig. 2(a). An example of a single cycle of the process waveform is shown in Fig. 2(b). The average coating thickness for this sample, measured using the eddy current gauge, was $8.4 \pm 1.3 \mu \mathrm{m}$.

\subsection{Optical emission spectra}

All spectra were obtained concurrently with small area DC testing of samples. A broad spectrum, taken with a 300 line grating and a
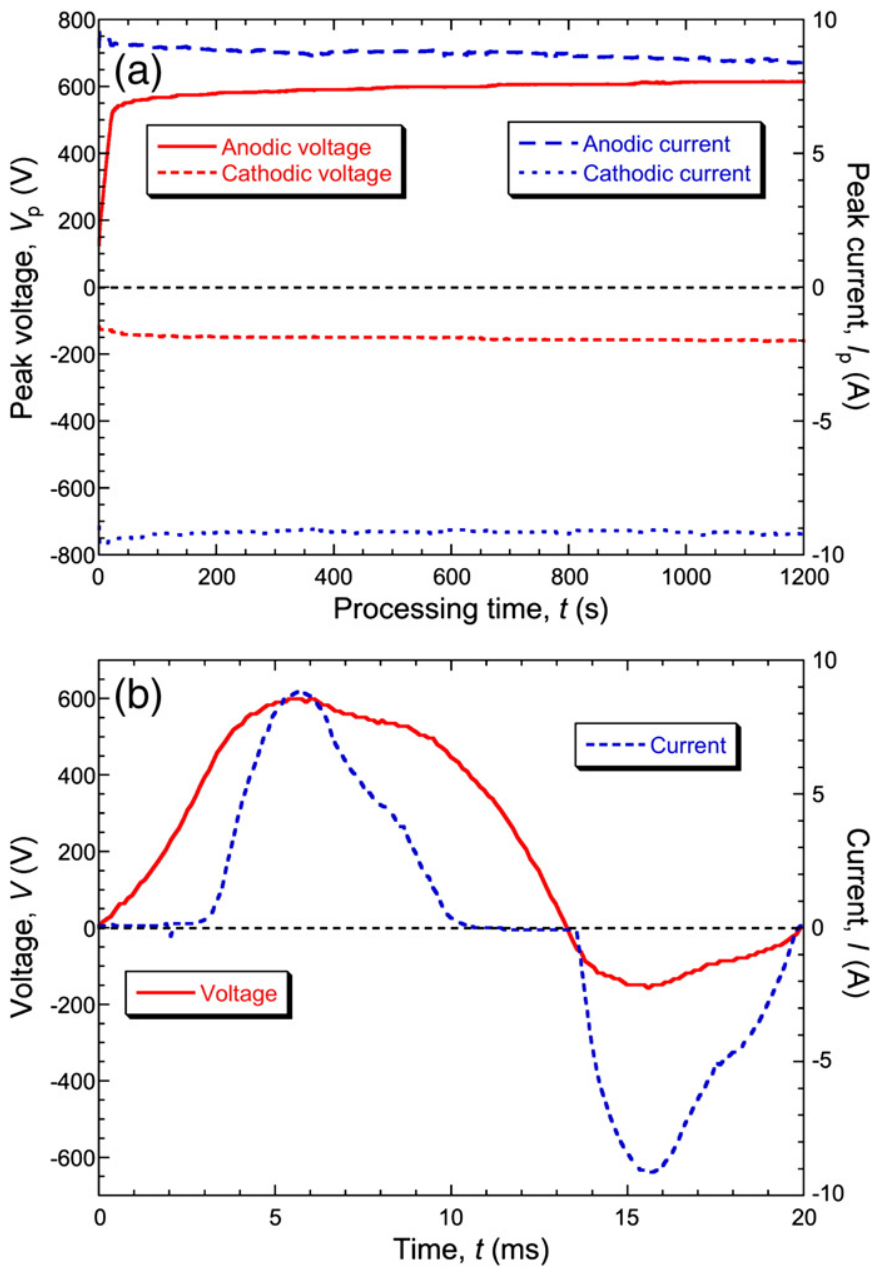

Fig. 2. (a) Typical variation of the peak anodic and cathodic voltage and current during the first 20 min of PEO processing and (b) voltage and current waveforms during a single cycle, after $10 \mathrm{~min}$ of processing. 


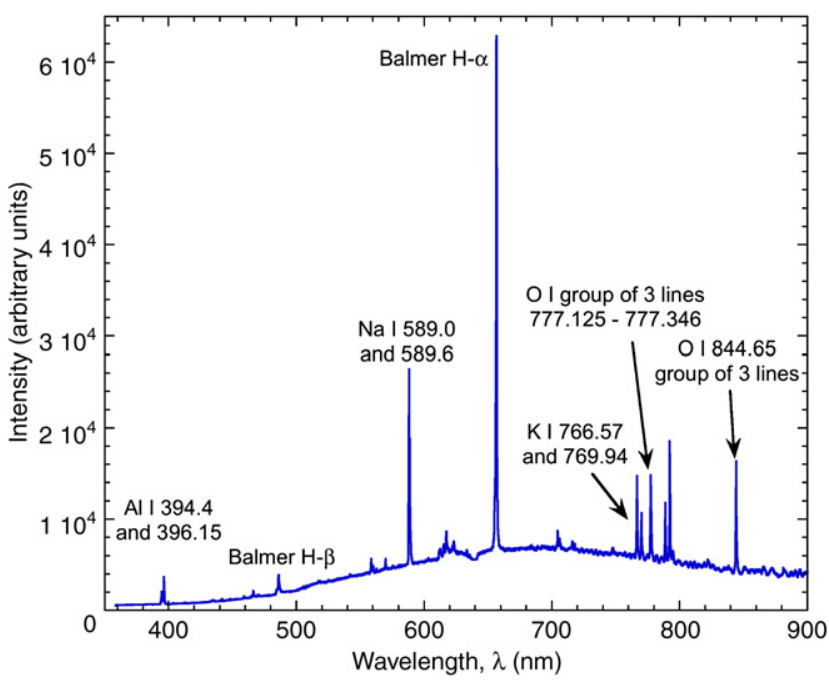

Fig. 3. Typical emission spectrum from PEO discharges, taken with the 300 line grating.

resolution of $0.2 \mathrm{~nm}$, is presented in Fig. 3. Strong emission lines are seen for atomic $\mathrm{O}, \mathrm{Na}$ and $\mathrm{K}$ (from the electrolyte), and for $\mathrm{Al}$ (from the substrate). Strong peaks are also seen corresponding to hydrogen $\alpha$ and $\beta$ Balmer lines.

Emission lines from substrate elements and ionized species were observed at lower intensities and at lower wavelengths. High resolution spectra, taken with the 1800 line grating (resolution of $0.03 \mathrm{~nm}$ ) are presented in Fig. 4(a) and (b). The atomic or ionic species responsible for each line are indicated on the plots. A mixture of components from both substrate and electrolyte is seen. Additional information about the electronic transitions giving rise to the emission lines is given for some ionic species. Emission lines corresponding to molecular $\mathrm{OH}$ radicals were also observed with the 1800 line grating. The relevant spectral region is presented in Fig. 4(c).

\subsection{Small area $D C$ testing}

Raw current and voltage data, recorded at $1 \mathrm{MHz}$ sampling rate over a period of $400 \mathrm{~ms}$, are presented in Fig. 5(a). It can be seen that there are many clusters of current peaks, separated by periods with a low baseline current. Each cluster is accompanied by a decrease in the voltage across the sample, which recovers during the periods of low current, until another series ("cascade") of current peaks is initiated. This voltage drop is a deliberate consequence of the low maximum power output of the high voltage supply, which has a high internal resistance, causing the voltage to drop under load, and depletion of charge on the capacitor.

One of the cascades of current pulses is shown on a magnified time scale in Fig. 5(b). The discrete nature of the individual current pulses is evident here; some appear as the superposition of two peaks, and these probably represent a second event initiating elsewhere, whilst the first is still in operation. Many characteristics may be obtained from these data. However, an important requirement is to identify the beginning and end of each current pulse. The way in which this was tackled is illustrated in Fig. 5(c). The continuous plot represents experimental current data. The line with markers refers to a logical index variable evaluated automatically by a data processing programme. This was implemented using an algorithm based on a combination of local and global thresholds on the current, and the 4th order finite difference gradient of the current. An index value of 1 indicates the start of a significant peak in the current, while 0.5 indicates that the current has fallen to the baseline level. A value of 0.75 indicates that the current has dropped to less than $10 \%$ of the most recent peak current. This was introduced to avoid artefacts due to peaks separated by a low, but not baseline, plateau in the current.
Discharges were considered to initiate when the index reached 1 and terminate when it became 1 again, or changed to 0.5 or 0.75 .

Once current pulse start and end points had been indexed and listed, characteristics of individual discharges, and statistical data concerning large numbers of them, could be obtained. In the present work, the focus is on (a) the peak power delivered by the external circuit during each current pulse, (b) durations, and temporal separations, of current pulses, (c) total energy in each pulse and (d) total charge passing through the system during each pulse. Histograms of these characteristics are presented in Fig. 8.

\subsection{In-situ discharge monitoring during $50 \mathrm{~Hz}$ AC processing}

Typical voltage and current data from in-situ monitoring are presented in Fig. 6. These data include correction for LR decay, which
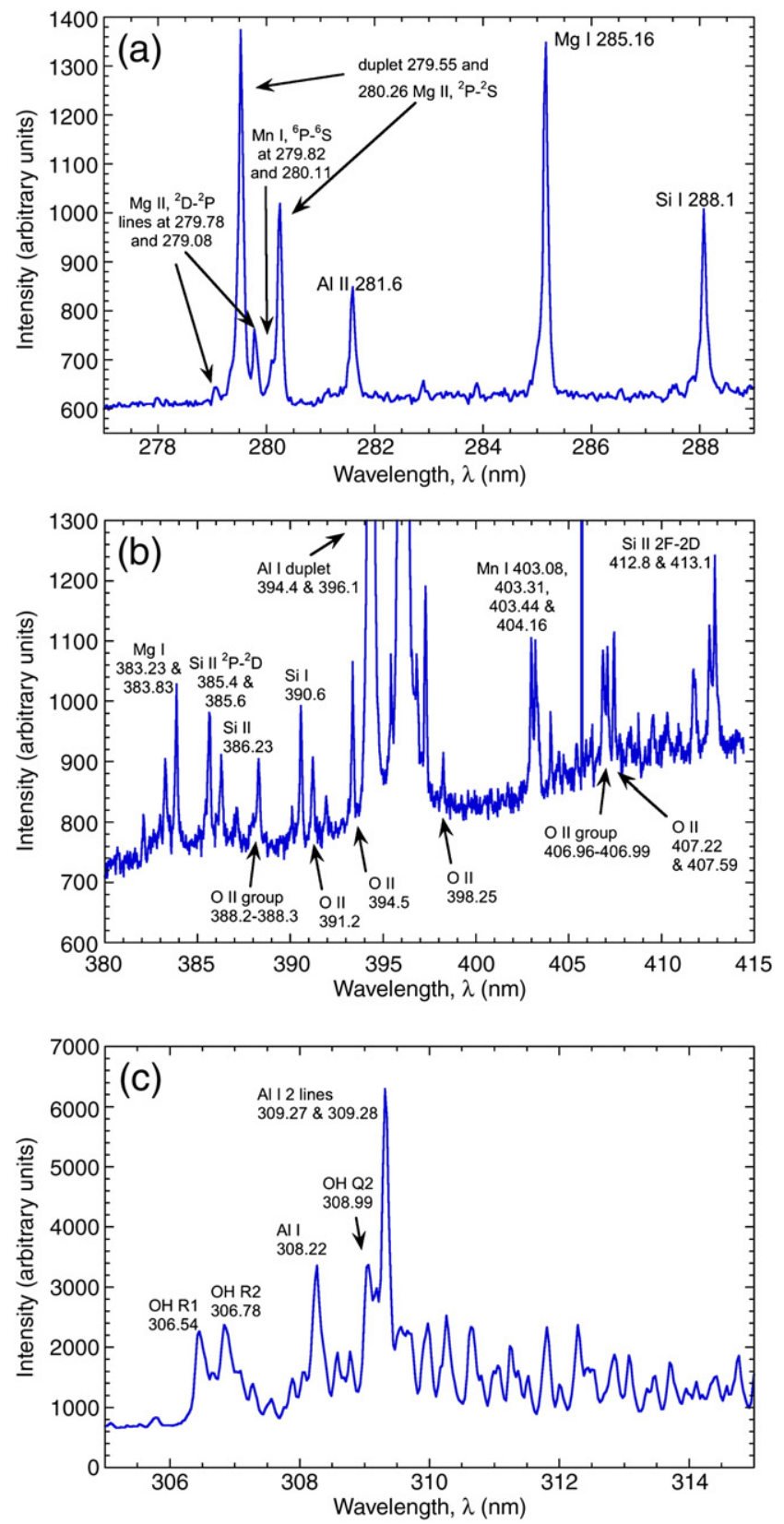

Fig. 4. High resolution spectra from typical PEO discharges, showing wavelength ranges containing peaks from: (a) $\mathrm{Mg}$ and $\mathrm{Mn}$, (b) $\mathrm{Mg}, \mathrm{Si}, \mathrm{Mn}, \mathrm{Al}$ and $\mathrm{O}$, and (c) $\mathrm{OH}$ (molecular rotation peaks). The $\mathrm{Si}, \mathrm{Mg}, \mathrm{Mn}$ and $\mathrm{Al}$ species in the plasma probably originate from the substrate, while the $\mathrm{O}$ and $\mathrm{OH}$ come from the electrolyte. 

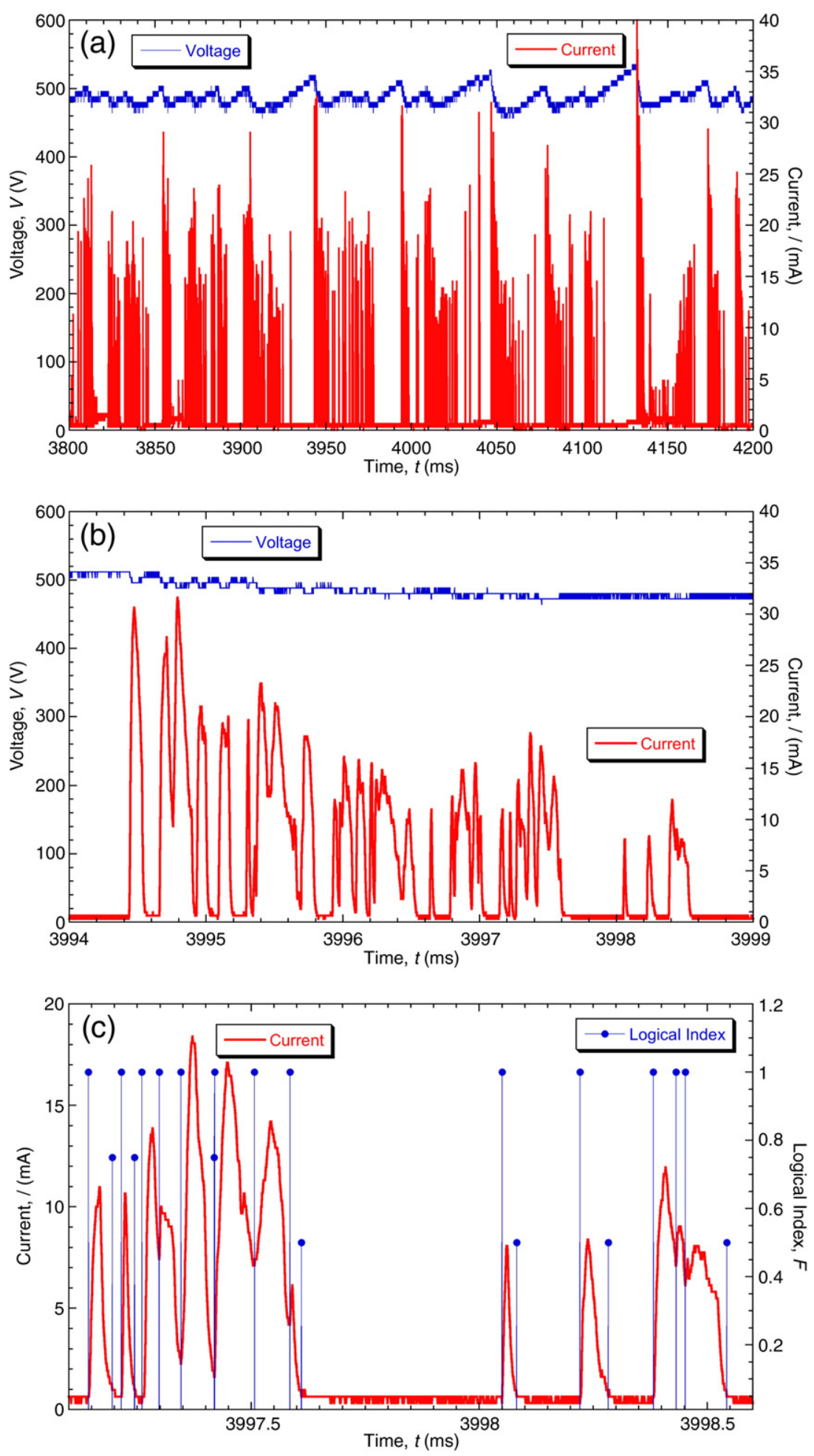

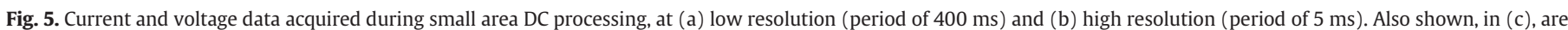
data for the indexing of discharge events, covering a period of about $1.5 \mathrm{~ms}$.

is a consequence of measuring pulses using a non-contact current transformer probe. The correction was made by measuring the response of the current probe to current pulses, which were simultaneously monitored via the potential drop over a known resistance. These calibration measurements were made using the small area DC testing equipment, with the current probe test loop inserted as the connection between the test resistor and the counterelectrode wire. The same loop as was used for the in-situ measure- ments was employed to collect the calibration data to minimise differences in any stray inductance. The uncorrected data can also be seen in Fig. 6. It may also be noted that no discharges were detected during the cathodic part of the cycle.

Calibration involved generating short groups of current pulses, then treating the group of pulses as a wave-packet. The discrete Fourier transforms of both the actual current (measured from the voltage over a known resistance), and the signal measured by the 


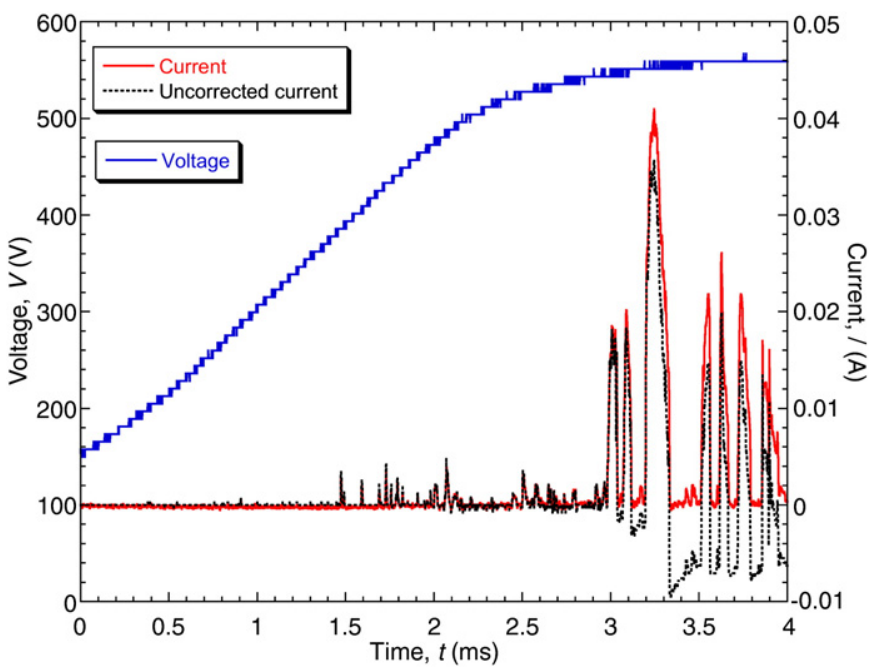

Fig. 6. Example of data from in-situ discharge monitoring during AC processing, taken between 19 and 21 min into processing. The discrete nature of the current response is clear, with small events giving way to larger current pulses as the applied voltage is increased. Qualitative agreement may be noted with the form of discharges observed in Fig. 5.

current probe, were obtained. One hundred such measurements were averaged to produce a discrete response function, for use in converting the signals from in-situ monitoring data to actual currents. The response function was also verified by applying it to ten segments of calibration data, which were not used in compilation of the response function. This system was found to be capable of allowing the signal from the current probe to be converted into the signal from monitoring the voltage over the resistance (minus a DC offset), to within an error never more than $1 \mathrm{~mA}$ and typically much less.

Once corrected for the LR decay, these data can be analysed in a similar fashion to the data from small area DC testing. The peak power, durations, charge and energy transferred during each current pulse were measured. Histograms are plotted in Fig. 8, on the same axes as the property data from small area DC testing. The in-situ data presented were collected between 19 and $21 \mathrm{~min}$, in $10 \mathrm{~s}$ segments.

These in-situ data are included here to give confidence in the relevance of small area DC testing to conventional, industrial scale $50 \mathrm{~Hz}$ PEO processing. Compilation of a full dataset concerning discharge properties, and variations in these as AC processing progresses, as well as correlation with microstructural features, is ongoing work and will be the subject of a future publication.

\subsection{Photomultiplier module data}

Fig. 7 shows an example of the current record from small area DC testing, along with the concurrently measured signal from the PMT module. The only information obtainable from these measurements is that the optical emissions from the discharge events correlate very closely with the associated current pulses. Of course, this is important information, confirming that individual events are being isolated and detected, both optically and electrically, with clear correlation between the two sets of measurements. However, attempting to infer anything from the level of the PMT signal during individual events is difficult, since no data are available on the precise optical path between any given event and the PMT module window.

\section{Interpretation of data}

\subsection{Optical emission spectra}

If local thermodynamic equilibrium (LTE) is assumed, then the relative total (integral) intensities of spectral lines of the same element, coming from transitions $m \rightarrow a$ and $n \rightarrow b$, can be related by the expression [26,27]

$\frac{J_{m a}}{J_{n b}}=\frac{g_{m} A_{m a}}{\lambda_{m a}} \frac{\lambda_{n b}}{g_{n} A_{n b}} \exp \left(\frac{E_{n}-E_{m}}{k_{B} T_{e}}\right)$.

In this equation, $T_{e}$ is the electron temperature, $\lambda_{m a}$ is the line wavelength, $A_{m a}$ is the spontaneous emission probability of the transition $m \rightarrow a, g_{m}$ and $E_{m}$ are respectively the statistical weight and energy of the upper level $m$ and $k_{B}$ is the Boltzmann constant. Values of $g$ and $A$ for different transitions are tabulated in the CRC Handbook [25].

It was suggested by Klapkiv [9] that there are several distinct zones, including a "core" plasma channel and a surrounding gas bubble. Emissions from hydrogen and the recombination continuum are postulated to come mostly from low density plasma in bubbles. The bubbles are formed in water as a result of plasma expansion, and possibly simultaneous gas evolution and combustion, and would be expected to exhibit lower electron densities and plasma temperatures.

In the current work, the intensity ratio for the Balmer $H_{\alpha}(656.3 \mathrm{~nm})$ and $H_{\beta}(486.1 \mathrm{~nm})$ lines was measured to be approximately 24 (see Fig. 3). When substituted into Eq. (1), this yields $T_{e} \sim 0.3 \mathrm{eV}(\sim 3500 \mathrm{~K})$. This is indeed a low plasma temperature. The intensity of the recombination continuum at high photon energies $\omega$ (low wavelengths) is [5] proportional to

$J(\omega)=\exp \left(\frac{-h \omega}{k_{B} T}\right)$

The inverse slope of the natural logarithm of $J(w)$ gives a similarly low temperature $\left(T_{e} \sim 0.3 \mathrm{eV}\right)$.

The full width at half-maximum of the $H_{\beta}$ line is about $0.35 \mathrm{~nm}$. The manufacturer's value for the contribution from instrumental broadening is approximately $0.05 \mathrm{~nm}$. This precision was confirmed by the observed separation of close oxygen 3F-3D lines with different total angular momentum, $794.7168 \mathrm{~nm}$ and $794.7548 \mathrm{~nm}$. Since only a very small contribution is expected from Doppler broadening at $T$ $\sim 0.3 \mathrm{eV}$, this suggests that the electron density in the peripheral regions is $N_{e} \sim 10^{15} \mathrm{~cm}^{-3}$, using published tables [26] and analysis $[5,28]$ of the micro discharges. Based on the value of the absorption coefficient for water, as a function of wavelength [29], it can be estimated that the effect on the Balmer line intensity ratio of passage

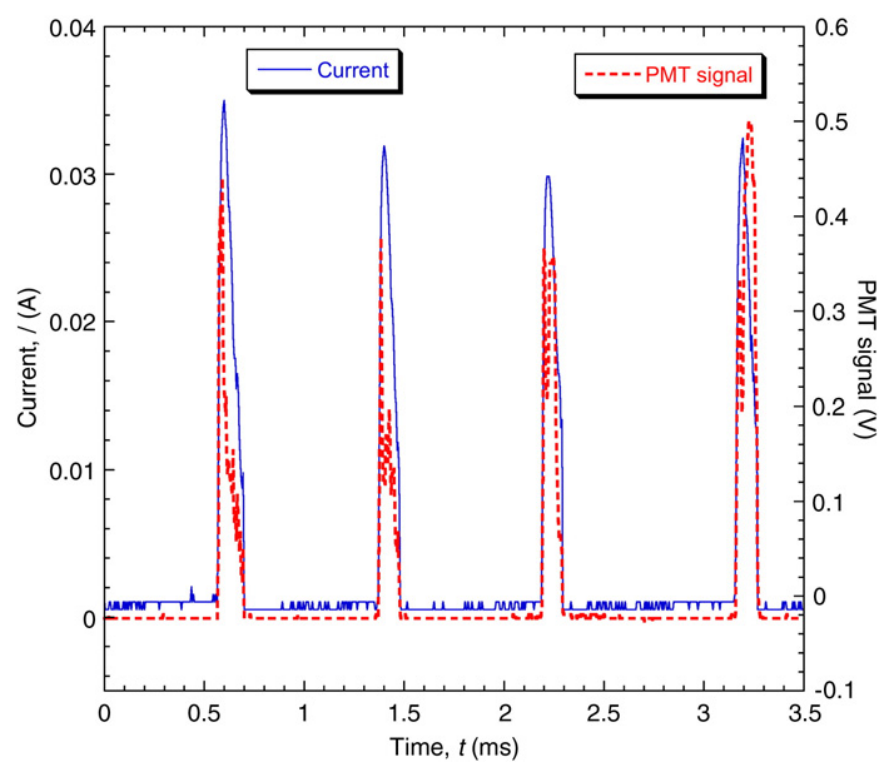

Fig. 7. Typical data for a sequence of current pulses, and for the associated light emission, obtained from a photomultiplier tube. 
through up to about $100 \mathrm{~mm}$ of water would be about $4 \%$, which is negligible for present purposes. Pressure broadening is also neglected in this analysis. Although the exact effect depends on the plasma composition, its order of magnitude can be estimated from previous studies [30,31]. The width $\gamma_{W}$, due to Van der Waals forces from $N_{0}$ neutral atoms, is given by

$\gamma_{W} \sim(1 \div 10) \cdot 10^{-20} N_{0}\left(\mathrm{~cm}^{-1} / \mathrm{cm}^{-3}\right)$

Using the Saha equations for ionisation equilibrium [26,27], and assuming mainly an aluminium-oxygen singly ionised plasma with $T_{e} \sim 0.3 \mathrm{eV}, N_{e} \sim 10^{15} \mathrm{~cm}^{-3}$ results in $N_{0} \sim 10^{18} \mathrm{~cm}^{-3}$ for the lower temperature plasma. In wavelength terms, Eq. (3) suggests $\gamma_{W} \sim$ $\left(10^{-3} \div 10^{-2}\right) \mathrm{nm}$ for transition at $500 \mathrm{~nm}$, which is negligible in comparison with the measured (mainly Stark) width. The analysis [26] is therefore valid.

It is worth noting that the estimated low temperature is in agreement with the observed emission of some electrolyte species at dilute concentration ( $\mathrm{Na}, \mathrm{K}, \mathrm{P}$ ). The only observed resonance lines for $\mathrm{Na}$ and $\mathrm{K}$ were $\sim 598 \mathrm{~nm}$ (corresponding to $E_{m} \sim 2 \mathrm{eV}$ ) and $\sim 766 \mathrm{~nm}$ (corresponding to $E_{m} \sim 1.6 \mathrm{eV}$ ), for these respective species. Emission from the next highest excited levels ( $404.4 \mathrm{~nm}, E_{m} \sim 3 \mathrm{eV}$ for $\mathrm{K}$, and $330.3 \mathrm{~nm}, E_{m} \sim 3.75 \mathrm{eV}$ for $\mathrm{Na}$ ) were not observed. This is partially due to smaller $A_{m a}$ values, but is mainly because of the large Boltzmann exponent at low $T_{e}$, see Eq. (1). Phosphorus lines have not been seen at all. This is unsurprising, since $P$ emission in $270-900 \mathrm{~nm}$ range comes from energy levels $E_{m}>9.5 \mathrm{eV}$ and therefore cannot be recorded at $T_{e} \sim 0.3 \mathrm{eV}$.

Silicon is present in both the substrate and the electrolyte. The observed Si emission comes from energy levels $E_{m}>5 \mathrm{eV}$. The above analysis implies that this emission must have come from the hotter plasma "core".

Charged ions are formed in the core of the plasma channel. In order to use Eq. (1) for analysis, both lines should ideally have high intensities and a reasonably large energy separation of the excited levels, $E_{m}$ and $E_{n}$. Absorption and scattering by the surrounding media could be important, which is why close lines are preferable. The $\mathrm{Mg}^{+}$lines ${ }^{2} \mathrm{P}-{ }^{2} \mathrm{~S}$, which are at $279.55 \mathrm{~nm}$ and $280.26 \mathrm{~nm}$, and the ${ }^{2} \mathrm{D}-{ }^{2} \mathrm{P}$ lines, at $279.78 \mathrm{~nm}$ and $279.08 \mathrm{~nm}$, are both suitable, although some of the lines are overlaid by the neutral Mn lines ${ }^{6} \mathrm{P}-{ }^{6} \mathrm{~S}$ at $279.82 \mathrm{~nm}$ and $280.11 \mathrm{~nm}$ (see Fig. 4(a)). This decreases the confidence with which the plasma temperature can be estimated, but a reasonable range in this case would be $T_{e} \sim 1.4 \pm 0.3 \mathrm{eV}$ $(\sim 16,000 \pm 3500 \mathrm{~K})$. Lines from singly-ionised silicon $\mathrm{Si}^{+}$were used to cross-check the result: The ${ }^{2} \mathrm{P}{ }^{2} \mathrm{D}$ lines at $385.4 \mathrm{~nm}$ and $385.6 \mathrm{~nm}$ were compared with ${ }^{2} \mathrm{~F}-{ }^{2} \mathrm{D}$ at $412.8 \mathrm{~nm}$ and $413.1 \mathrm{~nm}$. The outcome of this was $T_{e} \sim 1.5 \mathrm{eV}$ (see Fig. 4(b)), which is in good agreement.

The concentration of electrons in the core of the plasma was assessed by study of the broadening of $\mathrm{Mg}^{+}$and $\mathrm{Si}^{+}$lines. Electron impact widths, and corrections to the line profiles for given ions, were calculated using tables [26]. The results indicate a much higher electron density in the core of the discharge: $N_{e} \sim 5 \times 10^{17} \mathrm{~cm}^{-3}$. It is worth mentioning here that analyzing the relative intensities of neutral atom lines, together with lines from ions, as suggested by Klapkiv et al. [5], could lead to errors in the present case, since these emissions could come from different plasmas (core and peripheral regions).

Finally, the assumption of LTE in the plasma should be critically assessed. The electron density of the plasma core is $\sim 5 \times 10^{17} \mathrm{~cm}^{-3}$. Such a density is high enough to satisfy the LTE criterion for complex atoms $[5,32,33]$. According to theory, for a plasma to be at LTE, the electron density $N_{e}\left(\mathrm{~cm}^{-3}\right)$ should satisfy the requirement

$N_{e}\left(\mathrm{~cm}^{-3}\right)>10^{18}\left[T_{e}(\mathrm{eV}) / E_{\text {ion }}(\mathrm{eV})\right]^{1 / 2}\left[\Delta E(\mathrm{eV}) / E_{\text {ion }}(\mathrm{eV})\right]^{3}$

where $\Delta E(\mathrm{eV})$ is the largest electronic transition considered, $E_{\text {ion }}$ is an ionisation potential for the corresponding element and $T_{\mathrm{e}}$ is the electron temperature. In the measurements being made in the current work, the largest transition corresponds to the $\mathrm{Mg}$ and $\mathrm{Al}$ lines at $\sim 280 \mathrm{~nm}(\sim 4.5 \mathrm{eV})$ with a minimum ionisation potential $E_{\mathrm{ion}}(\mathrm{Al})$ $\sim 6 \mathrm{eV}$. Thus, the minimum electron density required for LTE is about $2 \times 10^{17} \mathrm{~cm}^{-3}$, which is below the inferred level for the core. On the other hand, for the low density plasma in the bubbles, the conditions $[5,26]$ for LTE are not satisfied. Nevertheless, excited energy levels
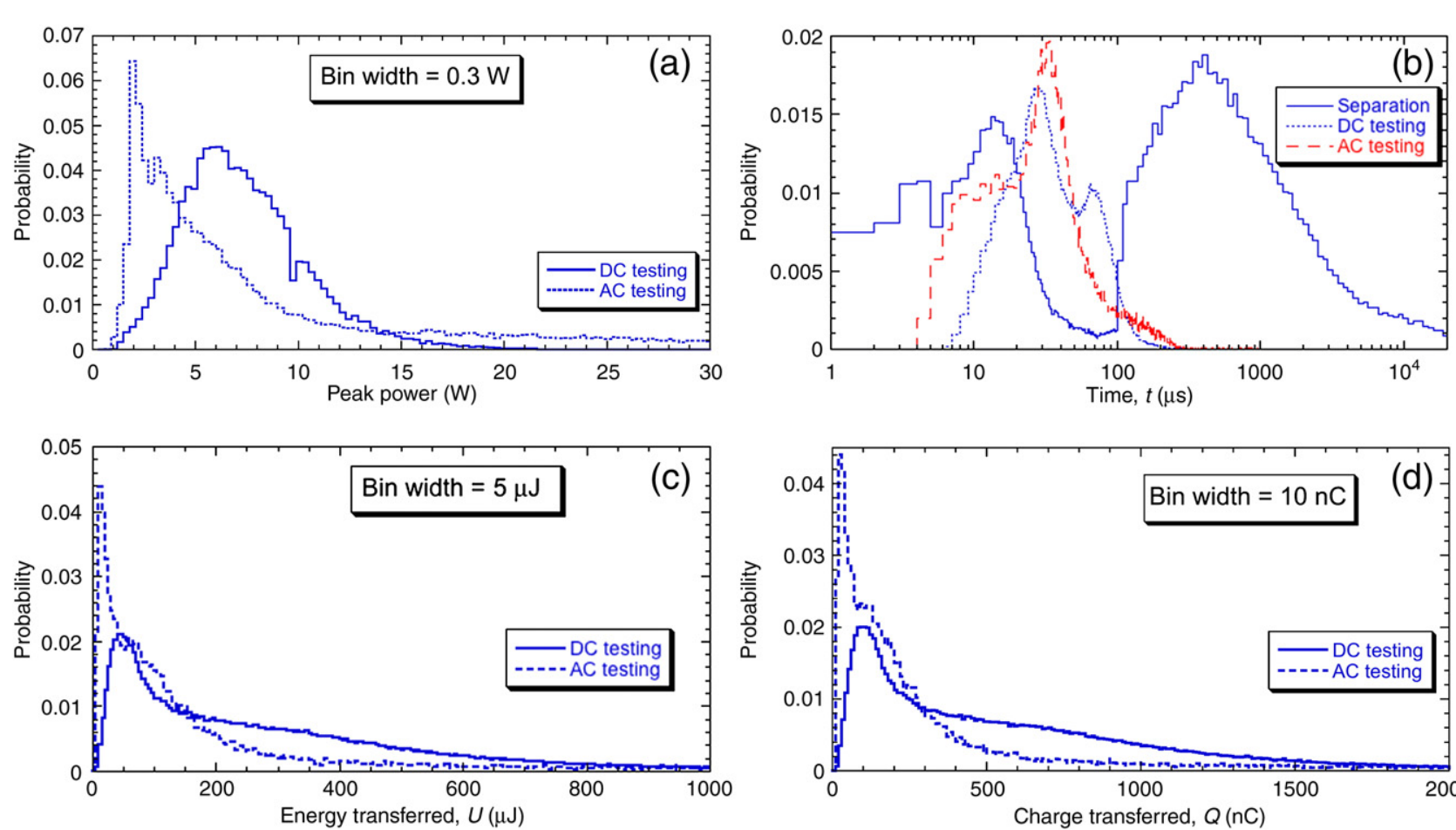

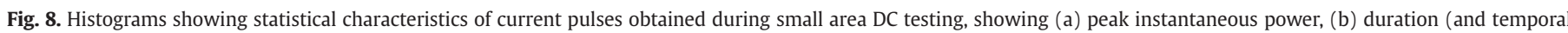
separation), (c) energy and (d) charge. 
could $[32,33]$ be in partial LTE with each other (but not with the deep energy levels). For example, considering only the $H_{\alpha}(3 \rightarrow 2)$ and $H_{\beta}(4 \rightarrow 2)$ lines, with $\Delta E \sim 3 \mathrm{eV}$ and $E_{\text {ion }}=13.6 \mathrm{eV}$ for hydrogen in Eq. (4), partial LTE for these hydrogen states does approximately exist for $N_{e} \sim 10^{15} \mathrm{~cm}^{-3}$, so the above estimate of the temperature is therefore valid.

A further estimate of the plasma temperature may be obtained from the relative intensities of $\mathrm{OH}$ molecular rotation lines, using a procedure outlined by de Izarra [34], which involves the relative intensity of $\mathrm{OH} \mathrm{R1,} \mathrm{R2} \mathrm{and} \mathrm{Q2} \mathrm{lines} \mathrm{at} \mathrm{306.537,} 306.776$ and $308.986 \mathrm{~nm}$ respectively. The relevant region of the spectrum is shown in Fig. 4(c). Two significant lines from atomic $\mathrm{Al}$ are also seen in this spectral region. The ratios $\mathrm{R} / \mathrm{Q}$ are about 0.6 , which corresponds to approximately $3000 \mathrm{~K}$ [34]. Assuming LTE, this should also be the plasma temperature. The value is close to the estimate of $0.3 \mathrm{eV}$ $(\sim 3500 \mathrm{~K})$ obtained from the hydrogen emissions.

\subsection{Electrical characteristics}

As can be seen in Fig. 5(a), the current response to the applied voltage takes the form of a series of clusters, or cascades, of pulses. A single cascade is shown at higher resolution in Fig. 5(b). Cascades typically last for several ms, and are separated by several ms of low background current, when there are presumably no dielectric breakdown events. This baseline current may be due to ohmic conduction through the coating. The data suggest that the DC resistance of the coating is $\sim 1.28 \pm 0.35 \mathrm{M} \Omega$ when no discharges are taking place. Converting this to a resistivity (using the measured thickness of $8.4 \pm$ $1.3 \mu \mathrm{m}$ and noting that the diameter of the test well was $6 \mathrm{~mm}$ ) gives a value of $1.37 \pm 0.43 \mathrm{M} \Omega \mathrm{m}$.

The statistical data for the peak instantaneous power in a current pulse, during DC testing (Fig. 8(a)), shows a strong peak around $6 \mathrm{~W}$, and extends up to $20 \mathrm{~W}$. The median power is $\sim 6.5 \mathrm{~W}$. The fraction of this power responsible for resistive heating in the electrolyte is difficult to determine, since the dimensions of the plasma are not accurately known, and will change over the course of the discharge development. The data from AC processing, after about $20 \mathrm{~min}$, show good agreement with the DC testing data, although there are some differences. In particular, the AC processing reveals a greater fraction of smaller scale events, leading to the peak in the distribution occurring at a lower value of about $2 \mathrm{~W}$. The large events do, however, reach higher peak currents, and occur at higher voltage, leading to the falling edge of the peak power distribution extending to higher power levels in the AC case. The largest observed was 64.7 W peak instantaneous power. The median value for the AC processing was $\sim 5.3 \mathrm{~W}$

The data for the pulse duration in DC testing (Fig. 8(b)) show two peaks, one around 30-40 $\mu$ s and a second at around 60-70 $\mu$ s. There were some pulses ( $\sim 1 \%$ of the total) with durations above $200 \mu \mathrm{s}$, and very occasional ones above $1 \mathrm{~ms}$. Whether such extended pulses correspond to discrete electrical breakdown and plasma formation, or to re-anodising after particularly destructive events, is at present unclear. The median pulse duration was $\sim 52 \mu \mathrm{s}$. The data from AC processing again show good correlation with those from DC testing, with a main peak between 30 and $40 \mu \mathrm{s}$ and a median value of $\sim 42 \mu \mathrm{s}$. It is clear from Fig. 8(b), however, that the fraction of very short lifetime events is greater during AC processing than in DC testing. This cannot be due to the alternating nature of the current, since the frequency is too low to be a major factor on microsecond timescales. It could be caused by events during AC processing being in competition for current with many other events occurring concurrently in neighbouring regions.

The data for temporal separation of current pulses (see Fig. 8(b)) show two distinct peaks at 13 and $400 \mu$ s, then a slight hump at around $10 \mathrm{~ms}$ and very few beyond $35 \mathrm{~ms}$. The peaks at 13 and $400 \mu$ s probably represent typical separations of pulses within cascades (Fig. 5(b)), with the region above $1 \mathrm{~ms}$ corresponding to periods between the end of one cascade and the start of the next (Fig. 5(a)).

\subsection{Correlation with optical imaging work}

These very short durations and separations of individual current pulses raise several issues. In a typical coating production system, with a relatively large surface area being treated, there will be many discharge events occurring simultaneously over the surface at any given time. Monitoring of the current during such macroscopic processing, irrespective of the data acquisition rate, cannot reveal information about the duration, or other characteristics, of individual discharges. Some previous work has employed video imaging of a selected area, in an attempt to bypass this problem. However, with such techniques, the duration of each exposure is usually relatively long - at least about $10 \mu$ s, and often much longer. Of course, there is a serious problem of insufficient light being emitted to form an image if the exposure time is very short, and in this case the difficulty cannot be resolved by using intense external light sources. Therefore, if the temporal characteristics being reported here are correct, then it is very unlikely that optical imaging techniques could be effectively used to obtain reliable information about them.

It seems likely that the current pulses which make up each cascade (see Fig. 5(b)) are occurring in the same physical location, or at least in the immediate vicinity. It may also be the case that further cascades occurring shortly afterwards are more likely to take place in such locations. Possible explanations for such an effect could include the site retaining residual heat, or being "primed" in some other way for further discharge by offering a low resistance path. In an attempt to correlate results obtained in the present work with the video imaging studies of Matykina et al. [3], the data presented in Fig. 8(b) for DC testing were re-analysed using time bins of $10 \mathrm{~ms}$, which was the exposure period employed by them [3]. The number of current pulses in each 10 ms period was counted and the lifetime of an "event" taken to be the time difference between one $10 \mathrm{~ms}$ period with no current pulses and the next one with no pulses. The outcome of this operation is shown in Fig. 9, together with data of Matykina et al. [3]. It can be seen that there is good general agreement, although it should be noted that the video data relate to studies on $\mathrm{Ti}$, while the present work concerns only Al. It should also be noted that the clusters recorded in the present work were generally of shorter duration than $10 \mathrm{~ms}$, so the assumption made, for the purposes of this comparison, that all pulses in each time bin were located at or near the same location will not be exact. Nevertheless, the data do suggest that the cascades being detected in the current work are likely to be recorded in video imaging experiments as single events occurring at single sites.

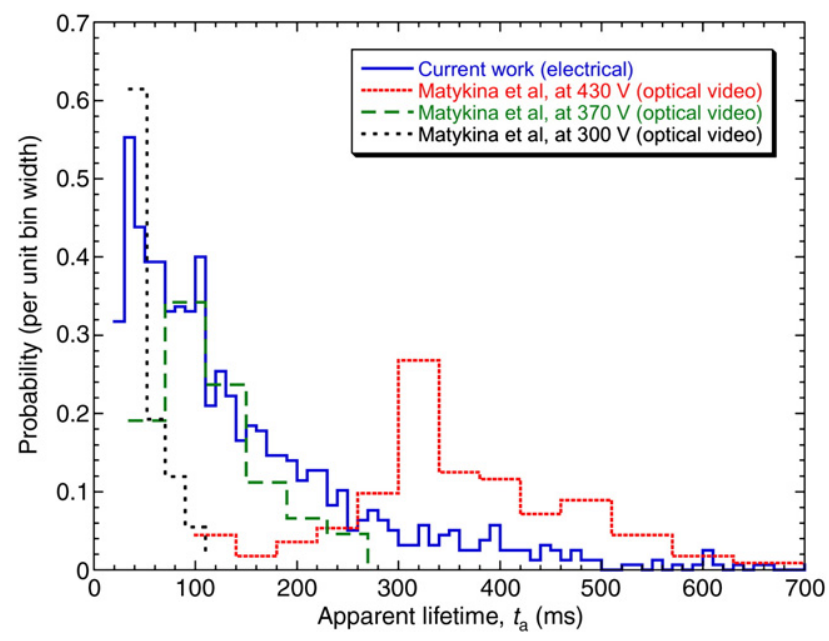

Fig. 9. Histograms showing statistical characteristics for the apparent lifetimes of (cascades of) current pulses, obtained in the present work during small DC testing, using a bin width of $10 \mathrm{~ms}$, and also data from the video imaging work of Matykina et al. [3], showing results obtained using an image exposure time of $10 \mathrm{~ms}$. 
In a very recent video imaging study, using an exposure time of $50 \mu \mathrm{s}$, Arrabal et al. [7] found that the lifetimes of the shortest events were in the approximate range of $50-185 \mu \mathrm{s}$, although obviously they recognised that they'd be unable to record lifetimes of less than $50 \mu$ s. These data correspond quite well with current pulse lifetimes found in the present work. It is worth noting, however, that the data in Fig. 8(b) suggest that, even with $50 \mu$ s exposure periods, it would be possible to capture several current pulses in a single frame, assuming that they were occurring at the same location. The estimate of average discharge lifetime obtained earlier by Van et al. [12] using electrical methods was $170 \mu$ s, which corresponds well with both the observations of Arrabal et al. [7] and the present work (Fig. 8(b)). The magnitude of typical discharge pulse currents obtained by Van et al. [12] was $~ 70 \mathrm{~mA}$, which is also similar to values obtained in the present work, of up to about $35 \mathrm{~mA}$ (Fig. 5).

\subsection{Inferences about the mechanisms of coating growth}

The data presented here allow several conclusions to be drawn about the mechanisms of coating growth. The first is that the discharges are discrete in nature and very short-lived. It can also be seen from Fig. 7 that the optical emissions from discharge events correspond very closely with the current pulse associated with each event. This implies that each separate discharge event during PEO processing terminates when the plasma generated has expended and cooled, such that the electrons and atomic species recombine to form neutral species, disconnecting the site of the discharges from the circuit, at least temporarily.

Certainly the magnitudes of operating voltages during PEO processing, and the fact that they tend to remain approximately constant as the coating grows, suggest that the electric field necessary for conventional dielectric breakdown of the oxides concerned is being generated throughout the process across a very thin layer ( $1 \mu \mathrm{m}$ in thickness?). The presence in PEO coatings of substantial levels of inter-connected porosity [4], which is presumably full of electrolyte throughout processing, is also very suggestive.

At present, it's rather difficult to make any inferences based on the data presented here for the plasma density, and for the energy and charge transferred during single pulses. Correlation of these data with macroscopic growth rates and external electrical circuit characteristics can only be attempted once the number of events occurring per unit area per unit time is known. The parallel small area processing and monitoring technique outlined here should have the capability of providing such data, by studying several different sizes of small area, and work is ongoing in this direction.

\section{Conclusions}

The following conclusions can be drawn from this work.

(a) The electrical discharge events taking place during PEO processing have been investigated, using both conventional and small area experimental arrangements, with particular attention being paid to the optical emission spectra of the associated plasma and the duration and temporal distribution of the electrical current pulses.

(b) Individual discharge events are of short duration (tens to hundreds of $\mu \mathrm{s})$, but these pulses show a marked tendency to occur in clusters, or cascades, with short intervals (tens to hundreds of $\mu \mathrm{s}$ ) between each pulse and a cascade containing large numbers of pulses (tens or hundreds). Cascade durations thus typically range from a few ms up to a few tens of $\mathrm{ms}$ in DC testing, although they may be limited by the duration of anodic polarization in $\mathrm{AC}$ processing.

(c) There is strong circumstantial evidence that all of the pulses in a particular cascade are occurring in the same location, or at least in the immediate vicinity. This is relevant to a number of previous observations, for example using high speed video imaging techniques, in which it seems likely that such cascades may have been interpreted as single events.

(d) The plasma formed during a discharge event lasts only as long as the event itself, ie the duration of current flow, indicating very rapid rates of heating and cooling in the vicinity of a discharge.

(e) Plasma emission spectra are indicative of there being two distinct regions, a central core of high temperature $(\sim 16,000 \pm$ $3500 \mathrm{~K})$, with a high electron density $\left(N_{e} \sim 5 \times 10^{17} \mathrm{~cm}^{-3}\right)$, and a peripheral region, probably extending into the surrounding electrolyte, which is much cooler ( 3000-4000 K) and less dense $\left(N_{e} \sim 10^{15} \mathrm{~cm}^{-3}\right)$. Information has also been obtained about the species present in the plasma. It's clear that there is a complex mixture, incorporating species originating both in the substrate and in the electrolyte.

(f) Certain characteristics reported here, and also several features observed in previous studies, appear to be consistent with a mechanism in which pulse formation occurs by repeated dielectric breakdown through, and reformation of, a thin $(\sim 1 \mu \mathrm{m})$ oxide film at the root of the coating (interface with the substrate). In this model, the region above such a location would initially comprise ceramic with inter-connected porosity, filled with electrolyte (across which the potential drop would be small).

(g) Measured properties of electrical discharges range over several orders on magnitude in both DC testing and AC processing. It's probably sensible to view the discharges occurring during PEO processes as forming part of a continuous spectrum, instead of classifying different "regimes" of processing, in which different types of discharge occur.

\section{Acknowledgements}

Financial support has come from EPSRC via a studentship (for CSD) and a Platform Grant (for IOG). The authors are grateful for the extensive technical assistance of Dr. Keith Page, with electronic circuitry issues. A contribution was also made by John McCaffrey, of Princeton Instruments, whose collaboration concerning the spectroscopic work was extremely helpful. Experiments carried out in Cambridge as part of an undergraduate project undertaken by Hannah Brice and Kevin Heritage were of assistance in developing some of the techniques employed in the present work. The cooperation of Dr. Endzhe Matykina, of Manchester University, concerning the video imaging work being undertaken there, is also gratefully acknowledged.

\section{References}

[1] G. Sundararajan, L. Rama Krishna, Surf. Coat. Technol. 167 (2003) 269.

[2] J.A. Curran, T.W. Clyne, Surf. Coat. Technol. 199 (2005) 168.

[3] E. Matykina, A. Berkani, P. Skeldon, G. Thompson, Electrochim. Acta 53 (2007) 1987.

[4] J.A. Curran, T.W. Clyne, Acta Mater. 54 (2006) 1985.

[5] M. Klapkiv, H. Nykyforchyn, V. Posuvailo, Mater. Sci. 30 (1994) 333.

[6] F. Mécuson, T. Czerwiec, T. Belmonte, L. Dujardin, A. Viola, G. Henrion, Surf. Coat. Technol. 200 (2005) 804.

[7] R. Arrabal, E. Matykina, T. Hashimoto, P. Skeldon, G.E. Thompson, Characterization of AC PEO coatings on magnesium alloys. In preparation.

[8] M. Klapkiv, Mater. Sci. 31 (1995) 494.

[9] M. Klapkiv, Mater. Sci. 35 (1999) 279.

[10] M. Klapkiv, O. Chuchmarev, P. Sydor, V. Posuvailo, Mater. Sci. 36 (2000) 66

[11] A.L. Yerokhin, L.O. Snizhko, N.L. Gurevina, A. Leyland, A. Pilkington, A. Matthews, J. Phys., D. 36 (2003) 2110.

[12] T. Van, S. Brown, G. Wirtz, Am. Ceram. Soc. Bull. 56 (1977) 563.

[13] V. Kadary, N. Klein, J. Electrochem. Soc. 127 (1980) 139.

[14] N. Klein, V. Moscovici, V. Kadary, J. Electrochem. Soc. 127 (1980) 152.

[15] T. Distefano, M. Shatzkes, J. Vac. Sci. Technol. 12 (1975) 37.

[16] T. Distefano, M. Shatzkes, J. Vac. Sci. Technol. 13 (1976) 50.

[17] N. Klein, M. Albert, J. Appl. Phys. 53 (1982) 5840.

[18] N. Klein, Adv. Phys. 21 (1972) 605.

[19] N. Klein, J. Appl. Phys. 21 (1982) 5828.

[20] N. Klein, P. Solomon, J. Appl. Phys. 47 (1976) 4364

[21] I. Kashat, N. Klein, J. Appl. Phys. 48 (1977) 5217.

[22] X. Nie, E. Meletis, J. Jiang, A. Leyland, A. Yerokhin, A. Matthews, Surf. Coat. Technol. 149 (2002) 245 
[23] F. Monfort, A. Berkani, E. Matykina, P. Skeldon, G.E. Thompson, H. Habazaki, K. Shimizu, Corros. Sci. 49 (2007) 672.

[24] Y. Ralchemko, A. Kramida, J. Reader, NIST Atomic Spectra Database (version 3.1.5), National Institute of Standards and Technology, 2008.

[25] CRC, Handbook of Chemistry and Physics, CRC Press, Cleveland, 1976.

[26] H.R. Griem, Spectral line broadening by plasmas, Academic Press, New York, 1974

[27] H.R. Griem, Principles of plasma spectroscopy, Cambridge University Press, Cambridge, 1997.

[28] I.O. Golosnoy, Plasma Phys. Rep. 27 (2001) 497.
[29] S.A. Sullivan, Opt. Soc. Am. J. 53 (1963) 962.

[30] J.F. Kielkopf, J. Phys. B: Atom. Molec. Phys. 9 (1976) L547.

[31] M. Kotteritzsch, W. Griest, A. Hese, J. Phys. B: Atom. Molec. Phys. 25 (1992) 913.

[32] R.W.P. McWhirter, in: RH Huddlestone, SL Leonard (Eds.), Plasma Diagnostic Techniques, Spectral Intensities, Academic Press, New York, 1965, p. 201.

[33] H. Griem, Phys. Rev. 131 (1963) 1170.

[34] C. de Izarra, J. Phys., D, Appl. Phys. 33 (2000) 1697. 